\title{
1 The relationship between a deformation-based eddy parameterization and the LANS- $\alpha$ turbulence model
}

\author{
3 \\ Scott D. Bachman ${ }^{\mathrm{a}}$, James A. Anstey ${ }^{\mathrm{b}}$, Laure Zanna ${ }^{\mathrm{c}}$ \\ ${ }^{a}$ National Center for Atmospheric Research, Boulder, CO, USA \\ ${ }^{b}$ Canadian Centre for Climate Modeling and Analysis, Environment and Climate Change Canada, Victoria, British Columbia, Canada \\ ${ }^{c}$ Atmospheric, Oceanic, and Planetary Physics, Department of Physics, University of Oxford, Oxford, UK
}

\section{Abstract}

A recent class of ocean eddy parameterizations proposed by Porta Mana and Zanna (2014) and Anstey and Zanna (2017) modeled the large-scale flow as a non-Newtonian fluid whose subgridscale eddy stress is a nonlinear function of the deformation. This idea, while largely new to ocean modeling, has a history in turbulence modeling dating at least back to Rivlin (1957). The new class of parameterizations results in equations that resemble the Lagrangian-averaged Navier-Stokes- $\alpha$ model (LANS- $\alpha$, e.g., Holm et al. 1998a). In this note we employ basic tensor mathematics to highlight the similarities between these turbulence models using component-free notation. We extend the Anstey and Zanna (2017) parameterization, which was originally presented in $2 \mathrm{D}$, to $3 \mathrm{D}$, and derive variants of this closure that arise when the full non-Newtonian stress tensor is used. Despite the mathematical similarities between the non-Newtonian and LANS- $\alpha$ models which might provide insight into numerical implementation, the input and dissipation of kinetic energy between these two turbulent models differ.

Keywords: Eddy parameterization; deformation; LANS- $\alpha$; mesoscale; non-Newtonian fluid

\section{Introduction}

The problem of parameterizing ocean mesoscale eddies has received considerable attention in recent years. Many sophisticated closures have been developed which seek to move beyond the paradigm of purely downgradient tracer transport, and which appeal to the expected turbulent cascades of the large-scale flow. Both observations (Stammer, 1997; Wang et al., 2010; Callies and Ferrari, 2013; Rocha et al., 2016) and modeling studies (Klein et al., 2008; Capet et al., 2008; Sasaki and Klein, 2012; Rocha et al., 2016) indicate that large-scale ocean turbulence is quasi-geostrophic, featuring an upscale cascade of kinetic energy (Scott and Wang, 2005; Scott and Arbic, 2007) and a forward (downscale) cascade of potential enstrophy. The upscale energy cascade has frequently been a focus of recent parameterization methods because it implies an energy flux away from dissipative scales, thereby conflicting with the need to dissipate resolved kinetic energy to maintain numerical stability. This dissipation implies that the upscale cascade may be attenuated or arrested unphysically, and the resulting energy loss may be complicit in causing the large-scale circulation in global models to be weak compared to observations (e.g. Kjellsson and Zanna, 2017).

${ }^{*}$ Corresponding Author address: Scott Bachman, National Center for Atmospheric Research, 1850 Table Mesa Drive, Boulder, CO, USA 80305; E-mail: bachman@ucar.edu; Phone: +1 (303) 497-1346 
There is considerable debate about the optimal way to approach the problem of compensating for the unphysical energy loss at large scales, and it is unclear whether using scale- and flow-aware dissipation (e.g. Bachman et al., 2017; Pearson et al., 2017) is sufficient in this regard. Viscous dissipation in these models is both unavoidable and necessary, and mimicking the upscale energy cascade by re-injecting the dissipated energy at larger spatial scales is warranted. Deterministic and stochastic approaches (e.g. Frederiksen and Davies, 1997; Berloff, 2005; Duan and Nadiga, 2007; Kitsios et al., 2013; Grooms and Majda, 2013; Porta Mana and Zanna, 2014; Jansen and Held, 2014; Jansen et al., 2015; Zanna et al. 2017) have shown promise in modeling certain dynamics characteristic of upscale energy transfer, such as upgradient momentum fluxes and energy backscatter. A more recent class of eddy closures (e.g. Porta Mana and Zanna, 2014; Anstey and Zanna, 2017; Zanna et al., 2017), which are based on the idea that turbulent stresses may be modeled by assuming a non-Newtonian stress-strain relation (e.g Ericksen, 1956; Rivlin. 1957; Crow. 1968; Lumley, 1970; Meneveau and Katz, 2000), may also be capable of modeling these dynamics.

Another branch of literature pertaining to the Lagrangian-averaged Navier-Stokes-alpha (LANS- $\alpha$ ) model also addresses the issue of correcting the large-scale energy. LANS- $\alpha$ has proven to be a skillful turbulence model in both engineering- (Chen et al., 1998, 1999; Holm et al., 2005) and geophysical-scale flows (Holm and Nadiga, 2003; Hecht et al., 2008a b; Petersen et al., 2008). The latter experiments showed a remarkable energisation of the eddy and mean kinetic energy fields equivalent to doubling the model resolution (Petersen et al. 2008), thereby achieving a similar large-scale energy amplification similar to the newer closures mentioned above. For geophysical flows, this energisation occurs because LANS- $\alpha$ effectively shifts the kinetic energy injection scale to lower wavenumbers, helping to spur the onset of baroclinic instability (Holm and Wingate 2005). As such, this aspect of LANS- $\alpha$ is especially exciting for eddy-permitting ocean modeling.

An interesting property of the LANS- $\alpha$ model is that its governing equations (Foias et al. 2001) are actually variants of the equations for an incompressible, homogeneous fluid of second grade (Dunn and Fosdick, 1974; Dunn and Rajagopal, 1995), whose stress-strain relation was recently examined in detail by Anstey and Zanna (2017, hereafter AZ) as a candidate turbulence closure for large-scale ocean models. It can be shown that if the AZ closure is extended to include the "memory" term neglected in their analysis, one recovers a set of governing equations which exhibit similarities to those used in LANS- $\alpha$. For the ocean modeller, the mathematical similarity between AZ and LANS- $\alpha$ is obscured by the vastly different notation used in their respective analyses, and the fact that the literature dealing with non-Newtonian fluids is often outside the scope of oceanographic research. That the governing equations of these models have similarities is significant, for one might expect that key advantages of one model, such as the baroclinic energisation by LANS- $\alpha$ mentioned above, would thus be conferred by AZ as well; while the eddy geometry from AZ, based on (e.g. Waterman and Lilly, 2015), can be translated to the LANS- $\alpha$ model.

The purpose of this paper is to show the similarities and discrepancies between the LANS- $\alpha$ model, $\mathrm{AZ}$, and second-grade fluid equations using tensor notation as in AZ. The connection between LANS- $\alpha$ and second-grade fluids has been mentioned in previous literature (e.g. Foias et al., 2001; Marsden and Shkoller 2001, among others) but to the authors' knowledge was never derived explicitly. Here, by starting from the stress tensor for a second-grade fluid, we derive and explicitly show how the connection arises between the LANS- $\alpha$ model and the Rivlin-Ericksen stress, and the AZ closure, while also providing a synthesis of previous ideas. To allow a thorough comparison of the different turbulence models, we will extend the original AZ formulation to 3D and show the equations that would result if one were to follow their approach and break the second Rivlin-Ericksen stress tensor into "memory" and "deformation" parts. For brevity the Coriolis and external body forces are left out of these derivations, although they can be added back in without affecting any part of the analysis. 


\section{A brief discussion of second-grade fluids and LANS- $\alpha$}

An extensive body of literature exists which discusses the mathematics and physics of both non-Newtonian fluids and LANS- $\alpha$, whose scope deserves a far more thorough discussion than is possible here ${ }^{1}$ Here only a few key elements in the development of both are mentioned.

Much of the nomenclature used in discussing non-Newtonian fluids stems from continuum mechanics, and is intended to extend to general coordinate systems and moving frames of reference. Objects defined below which may have familiar names in the oceanographic literature, such as the strain rate tensor, $\mathbf{S}$, or vorticity tensor, $\mathbf{W}$, may instead be formally referred to as the rate of deformation tensor and spin tensor, respectively. Other functions of these tensors and their time derivatives often appear. To keep this derivation accessible, here we will restrict consideration to a Cartesian, Eulerian frame, with velocity vector $\mathbf{u}=$ $(u, v, w)$. The velocity gradient tensor is defined as

$$
\nabla \mathbf{u}=\left[\begin{array}{ccc}
u_{x} & u_{y} & u_{z} \\
v_{x} & v_{y} & v_{z} \\
w_{x} & w_{y} & w_{z}
\end{array}\right],
$$

and its symmetric and antisymmetric parts as,

$$
\mathbf{S}=\frac{1}{2}\left(\nabla \mathbf{u}+\nabla \mathbf{u}^{T}\right) \quad \text { and } \quad \mathbf{W}=\frac{1}{2}\left(\nabla \mathbf{u}-\nabla \mathbf{u}^{T}\right)
$$

where $\nabla \mathbf{u}^{T}$ refers to the transpose of (1). Additionally, we will assume the fluid is Boussinesq, allowing us to replace variable density, $\rho$, with a constant, $\rho_{0}$.

An incompressible second-grade fluid is a particular class of non-Newtonian Rivlin-Ericksen fluids of differential type (Rivlin and Ericksen, 1955), which are materials in which only a very short part of the deformation history has an influence on the stress. Mathematically, this simply means that the stress in Rivlin-Ericksen fluids is treated as a function of the velocity gradient and some number of its higher time derivatives. For a second-grade fluid, the stress tensor is the sum of all tensors which can be formed using up to two spatial derivatives of the velocity field, and can be written (Criminale et al., 1958; Coleman and Noll, 1960)

$$
\boldsymbol{\sigma}=-p \mathbf{I}+\mu \mathbf{A}_{1}+\alpha_{1} \mathbf{A}_{2}+\alpha_{2} \mathbf{A}_{1}^{2}
$$

Here $p$ is the thermodynamic pressure and $\mu, \alpha_{1}$ and $\alpha_{2}$ are material moduli and are properties of the flow rheology, with $\mu$ being the familiar dynamic viscosity. While cases where the moduli are treated as functions of the strain rate have been considered (e.g. Criminale et al., 1958), the rheology is generally assumed to be homogeneous so that the viscosity and other stress moduli are treated as constants. $\mathbf{A}_{1}$ and $\mathbf{A}_{2}$ are the first and second Rivlin-Ericksen tensors, which represent the lowest-order approximations of the deformation history:

\footnotetext{
${ }^{1}$ For an excellent retrospective on the theory of incompressible second-grade fluids, the reader is encouraged to consult Dunn and Rajagopal ( 1995). Likewise, an interesting exposition on the development of LANS- $\alpha$ from concept to turbulence closure can be found in Holm et al. 12005।.
} 


$$
\begin{aligned}
& \mathbf{A}_{1}=2 \mathbf{S} \\
& \mathbf{A}_{2}=\frac{D \mathbf{A}_{1}}{D t}+\nabla \mathbf{u}^{T} \mathbf{A}_{1}+\mathbf{A}_{1} \nabla \mathbf{u} .
\end{aligned}
$$

The operator

$$
D / D t=\partial_{t}+\mathbf{u} \cdot \nabla
$$

is the usual material derivative. The equations of motion for this system state that the acceleration of the fluid is equal to the divergence of the stress tensor,

$$
\frac{D \mathbf{u}}{D t}=\frac{1}{\rho_{0}} \operatorname{div} \sigma .
$$

Together with the additional thermodynamical constraints (Dunn and Fosdick, 1974)

$$
\mu \geq 0, \quad \alpha_{1}+\alpha_{2}=0, \quad \alpha_{1} \geq 0,
$$

we will show that the momentum equations for second-grade fluids take the general form

$$
\begin{aligned}
\frac{D \mathbf{v}}{D t}+\nabla \mathbf{u}^{T} \cdot \mathbf{v} & =-\frac{1}{\rho_{0}} \nabla P+v \nabla^{2} \mathbf{u}+\mathcal{F} \\
\mathbf{v} & =\left(1-\alpha \nabla^{2}\right) \mathbf{u} \\
\alpha & =\frac{\alpha_{1}}{\rho_{0}} .
\end{aligned}
$$

The Lagrangian derivative in (9), and in all subsequent expressions, remains as defined in (6). Here we have introduced the kinematic viscosity, $v=\mu / \rho_{0}$, and a rescaled stress modulus, $\alpha$, for brevity and assume that they are both constant and positive. $P$ is a modified pressure whose exact form depends on whether one chooses to neglect terms in the nonlinear stress $\mathbf{A}_{2}$, which is the scenario explored by AZ. $\mathcal{F}$ represents extra terms that also appear in the momentum equations when terms in $\mathbf{A}_{2}$ are neglected, as will be shown in Sections 3.3 and 3.4. When no terms in $\mathbf{A}_{2}$ are neglected $\mathcal{F}=0$.

A unique feature of (9) is the appearance of a second velocity, $\mathbf{v}$, which is related to $\mathbf{u}$ by a Helmholtz operator. The interpretation of this velocity is a crucial difference between second-grade fluids and LANS$\alpha$. In the context of second-grade fluids it is appropriate to think of the nonlinear stress $\mathbf{A}_{2}$ as introducing several extra terms into the momentum equations that are dependent on the stress modulus $\alpha$. Introducing $\mathbf{v}$ allows us to write these equations more succinctly but it is not physically meaningful in and of itself. LANS$\alpha$ considers the advecting velocity $\mathbf{u}$ to be a filtered version of $\mathbf{v}$, where the notion of filtering the velocity arises through the Lagrangian averaging procedure (Hecht et al., 2008a). The filter itself is represented by the Helmholtz operator relating $\mathbf{u}$ and $\mathbf{v}$, and acts to suppress small-scale structures in $\mathbf{v}$ (Foias et al., 2001). This inspires alternative names for $\mathbf{u}$ and $\mathbf{v}$ as the smooth and rough velocity, respectively (e.g. Hecht et al. 2008a b; Petersen et al. 2008).

Excluding the viscous term, (9) is identical to the three-dimensional form of the Camassa-Holm equation (Camassa and Holm, 1993), also known as the Lagrangian-averaged Euler- $\alpha$ (LAE- $\alpha$ ) equations (Holm et al. 1998a b; Bhat et al. 2005). The LAE- $\alpha$ interpretation of these equations has been arrived at from multiple 
angles, including Hamiltonian systems (e.g. Holm et al., 1998a b), ensemble averaging of the fluid energy (Marsden and Shkoller, 2003), and Generalized Lagrangian-Mean (GLM) theory (Andrews and McIntyre 1978; Holm, 1999, 2002; Bhat et al. 2005). The addition of viscosity to the LAE- $\alpha$ equations relabels them as LANS- $\alpha$ and enables their interpretation as a turbulence model. However, the mathematical form of LANS- $\alpha$ remains distinct from (9) because its dissipation is treated as a function of $\mathbf{v}$ instead of $\mathbf{u}$. The stronger form of dissipation induced by using $\mathbf{v}$ instead of $\mathbf{u}$ in $\alpha$-models may be justified both on theoretical grounds (e.g. Marsden and Shkoller. 2001) as well as by numerical experiments (e.g. Chen et al., 1998 1999).

Lastly, while LANS- $\alpha$ is similar to the second-grade fluid equations, the parameter $\alpha$ takes a different physical meaning in the two models. In the second-grade fluid model $\alpha$ is a property of the fluid, whereas in LANS- $\alpha$, and in Porta Mana and Zanna (2014), Anstey and Zanna (2017), and Zanna et al. (2017) it is a property of the flow dynamics. The appearance of the modified material derivative and the interpretation of $\alpha$ as a statistical correlation length (Grooms and Zanna, 2017) can be understood in the context of the GLM framework (e.g. Holm et al., 2005). Numerical implementations of the LANS- $\alpha$ model have generally assumed the correlation length $\alpha$ to be constant. While this assumption is not strictly necessary, using a variable $\alpha$ introduces extra terms into the filtering operator relating $\mathbf{u}$ and $\mathbf{v}$ (Petersen et al. 2008). Variations in $\alpha$ may be important near boundaries (Holm et al., 2003), but these considerations have not been explored.

\section{Derivation of governing equations for memory and deformation terms in second-grade fluids}

\subsection{Preliminaries}

We will first establish the basic tensor algebra rules needed to derive the different formulations of these models starting from the stress tensor ( 3 ) and equation of motion (7). These rules will initially be written using Einstein summation notation for clarity before reverting to the more succinct component-free form later. Subscripts $i$ and $j$ will be reserved to define components within the Einstein notation, and are not to be confused with subscripts indicating spatial $(x, y, z)$ and temporal $(t)$ partial derivatives appearing elsewhere. Here the use of the term tensor refers only to objects of second-order, not the more general definition that includes scalars, vectors, and higher-order objects.

In tensor algebra a vector, $\gamma$, can both left- and right-multiply a tensor, $\boldsymbol{\Lambda}$, according to the rules

$$
\boldsymbol{\Lambda} \cdot \boldsymbol{\gamma}=\Lambda_{i j} \gamma_{j} \quad \boldsymbol{\gamma} \cdot \boldsymbol{\Lambda}=\gamma_{i} \Lambda_{i j},
$$

where henceforth the first subscript will indicate the row index and the second subscript the column index. Tensor-vector multiplication will always be indicated here with a dot. Multiplication of two tensors, $\boldsymbol{\Lambda}$ and $\boldsymbol{\Gamma}$, follows the matrix multiplication rules from linear algebra,

$$
\mathbf{\Lambda} \boldsymbol{\Gamma}=\Lambda_{i k} \Gamma_{k j},
$$

and will not feature a dot. Another operation, the double contraction, takes two second-order tensors as arguments and returns a scalar,

$$
\Lambda: \Gamma=\Lambda_{i j} \Gamma_{i j}
$$


Lastly, the divergence operates along each row (i.e. the sum is taken across columns) of a tensor according to the rule

$$
\operatorname{div} \boldsymbol{\Lambda}=\partial_{j} \Lambda_{i j}
$$

\section{Note that the notation in (13) differs from that used in AZ, who used $\nabla \cdot \boldsymbol{\Lambda}$ to indicate the divergence of a tensor. There is no consensus in the mechanics and cosmology literature regarding the use of either "div" (e.g. Gurtin et al., 2010) or "nabla" (e.g. Dill, 2006; Gonzalez and Stuart. 2008) notation to denote the divergence of a second-order tensor, or whether this operation acts along the rows (e.g. Gonzalez and Stuart 2008; Gurtin et al., 2010) or columns (e.g. Dill, 2006). Here we use the "div" notation for the divergence because it is unambiguous; the nabla notation for such operations will not be used here to prevent confusion. \\ The rules above can be used to write the following useful identities,}

$$
\begin{aligned}
\operatorname{div}(\boldsymbol{\Lambda} \boldsymbol{\Gamma}) & =\boldsymbol{\Lambda} \cdot(\operatorname{div} \boldsymbol{\Gamma})+(\boldsymbol{\Gamma} \cdot \nabla) \cdot \boldsymbol{\Lambda}^{T} \\
\operatorname{div} \nabla \mathbf{u} & =\nabla^{2} \mathbf{u} \\
\operatorname{div} \nabla \mathbf{u}^{T} & =0 \\
\operatorname{div} \mathbf{S} & =\operatorname{div} \mathbf{W}=\frac{1}{2} \nabla^{2} \mathbf{u},
\end{aligned}
$$

where the relation (16) is established by noting that $\operatorname{div} \nabla \mathbf{u}^{T}=\nabla\left(u_{x}+v_{y}+w_{z}\right)=0$ by incompressibilty. We also note that the dot product notations on the right side of (14) formally follow the rules established in (10), with the nabla operator $\nabla=\left(\partial_{x}, \partial_{y}, \partial_{z}\right)$ acting as a vector in this context.

Finally, we distinguish the vector and tensor norms, which are, respectively,

$$
\|\boldsymbol{\gamma}\|=\left(\sum_{i} \gamma_{i}^{2}\right)^{1 / 2} \quad\|\boldsymbol{\Lambda}\|=\left(\sum_{i, j} \Lambda_{i j}^{2}\right)^{1 / 2} .
$$

\subsection{Decomposition of the nonlinear stress tensor}

Our starting point is the stress tensor for a second-grade fluid, (3), which after substituting (4), (5), and (8) can be written

$$
\begin{aligned}
\boldsymbol{\sigma} & =-p \mathbf{I}+2 \mu \mathbf{S}+2 \alpha_{1}\left[\frac{D \mathbf{S}}{D t}+\mathbf{S} \nabla \mathbf{u}+\nabla \mathbf{u}^{T} \mathbf{S}\right]-4 \alpha_{1} \mathbf{S}^{2} \\
& =-p \mathbf{I}+2 \mu \mathbf{S}+2 \alpha_{1}\left[\frac{D \mathbf{S}}{D t}+\mathbf{S W}-\mathbf{W S}\right]
\end{aligned}
$$

where (22) follows from (19) by use of (2) to write $\nabla \mathbf{u}=\mathbf{S}+\mathbf{W}$ and $\nabla \mathbf{u}^{T}=\mathbf{S}-\mathbf{W}$. By (7) the fluid acceleration is equal to the divergence of $\sigma$, which after substituting (17) is

$$
\operatorname{div} \boldsymbol{\sigma}=-\nabla p+\mu \nabla^{2} \mathbf{u}+2 \alpha_{1} \operatorname{div}[\underbrace{\frac{D \mathbf{S}}{D t}}_{\text {Memory }}+\underbrace{(\mathbf{S W}-\mathbf{W S})}_{\text {Deformation }}] .
$$


Here we can now identify the familiar thermodynamic pressure gradient and harmonic viscosity terms, as well as div (SW - WS) as the candidate eddy closure studied in AZ. We will first complete the derivation for the AZ parameterization (deformation) and its complementary (memory) part separately, before returning to the full expression in the square brackets.

\subsection{Deformation (AZ parameterization)}

This part of the derivation will consider the stress tensor

$$
\boldsymbol{\sigma}=-p \mathbf{I}+2 \mu \mathbf{S}+2 \alpha_{1}[\mathbf{S W}-\mathbf{W S}]
$$

where the term in square brackets is the $3 \mathrm{D}$ version of the $\mathrm{AZ}$ parameterization and $\alpha_{1}$ here is equivalent to $\kappa$ in their notation. Employing the identity (14) to expand the divergences of each product,

$$
\begin{aligned}
& \operatorname{div}(\mathbf{S W})=\frac{1}{2} \mathbf{S} \cdot \nabla^{2} \mathbf{u}+(\mathbf{W} \cdot \nabla) \cdot \mathbf{S} \\
& \operatorname{div}(\mathbf{W S})=\frac{1}{2} \mathbf{W} \cdot \nabla^{2} \mathbf{u}-(\mathbf{S} \cdot \nabla) \cdot \mathbf{W},
\end{aligned}
$$

and in the context of the full momentum equations we then obtain, after using the identities in (2) and replacing $\alpha_{1} / \rho$ with $\alpha$ as per (9),

$$
\begin{aligned}
\frac{D \mathbf{u}}{D t} & =\frac{1}{\rho_{0}} \operatorname{div} \boldsymbol{\sigma} \\
& =-\frac{1}{\rho_{0}} \nabla p+\nu \nabla^{2} \mathbf{u}+\alpha\left[(\nabla \mathbf{u} \cdot \nabla) \cdot \nabla \mathbf{u}-\left(\nabla \mathbf{u}^{T} \cdot \nabla\right) \cdot \nabla \mathbf{u}^{T}+\nabla \mathbf{u}^{T} \cdot \nabla^{2} \mathbf{u}\right]
\end{aligned}
$$

Adding the identity

$$
\nabla \mathbf{u}^{T} \cdot \mathbf{u}=\frac{1}{2} \nabla\|\mathbf{u}\|^{2}
$$

to both sides of (26) and moving $\nabla \mathbf{u}^{T} \cdot \alpha \nabla^{2} \mathbf{u}$ to the left side, this becomes

$$
\frac{D \mathbf{u}}{D t}+\nabla \mathbf{u}^{T} \cdot \mathbf{v}=-\frac{1}{\rho_{0}} \nabla\left(p-\frac{\rho_{0}}{2}\|\mathbf{u}\|^{2}\right)+v \nabla^{2} \mathbf{u}+\alpha\left[(\nabla \mathbf{u} \cdot \nabla) \cdot \nabla \mathbf{u}-\left(\nabla \mathbf{u}^{T} \cdot \nabla\right) \cdot \nabla \mathbf{u}^{T}\right]
$$

The derivation of the deformation part is completed by making use of the identities

$$
\begin{aligned}
(\nabla \mathbf{u} \cdot \nabla) \cdot \nabla \mathbf{u} & =\frac{1}{2} \nabla\|\nabla \mathbf{u}\|^{2} \\
\left(\nabla \mathbf{u}^{T} \cdot \nabla\right) \cdot \nabla \mathbf{u}^{T} & =\left[\begin{array}{l}
\mathbf{S}: \mathbf{H}(u) \\
\mathbf{S}: \mathbf{H}(v)
\end{array}\right] \equiv \mathbf{S}: \mathbf{H}(\mathbf{u})
\end{aligned}
$$

where $\mathbf{H}$ is the Hessian matrix, 


$$
H(\cdot)_{i j}=\frac{\partial^{2}(\cdot)}{\partial x_{i} \partial x_{j}} .
$$

Substituting (29) and (30) into (28), the momentum equations can now be written as

$$
\frac{D \mathbf{u}}{D t}+\nabla \mathbf{u}^{T} \cdot \mathbf{v}=-\frac{1}{\rho_{0}} \nabla\left(p-\frac{\rho_{0}}{2}\|\mathbf{u}\|^{2}-\rho_{0} \frac{\alpha}{2}\|\nabla \mathbf{u}\|^{2}\right)-\alpha \mathbf{S}: \mathbf{H}(\mathbf{u})+\nu \nabla^{2} \mathbf{u} .
$$

We note the overall similarity between (32) and the LANS- $\alpha$ momentum equations with harmonic dissipation, given by (e.g., Hecht et al., 2008a)

$$
\frac{D \mathbf{v}}{D t}+\nabla \mathbf{u}^{T} \cdot \mathbf{v}=-\frac{1}{\rho_{0}} \nabla\left(p-\frac{\rho_{0}}{2}\|\mathbf{u}\|^{2}-\rho_{0} \frac{\alpha}{2}\|\nabla \mathbf{u}\|^{2}\right)+v \nabla^{2} \mathbf{v},
$$

where $D / D t$ remains defined as in (6). The differences between (32) and (33) reside in: 1) the extra double contraction term, $\alpha \mathbf{S}: \mathbf{H}(\mathbf{u})$, on the right side of (32), 2) the fact that the viscous terms depend on different velocities, and 3) the different velocity being advected, $\mathbf{u}$ in (32) and $\mathbf{v}$ in (33). The double contraction term appears because we have split the deformation and memory parts, and is absent when both parts are combined (Section 4). It will be shown in Section 3.4 that the material derivative of $\mathbf{v}$ stems from including the "memory" part, and so is missing when only deformation is considered. As mentioned in Section 2, the correct dissipation operator for LANS- $\alpha$ is dependent on $\mathbf{v}$, not $\mathbf{u}$, which is due to the interpretation of that model as a Lagrangian-averaged representation of the turbulent fluid (e.g. Marsden and Shkoller, 2001).

Here we appeal to the LANS- $\alpha$ literature, key aspects of which are summarized succinctly in Hecht et al. (2008a), to interpret the appearance of terms in (33) which differ from the traditional primitive equations. One of the fundamental features of LANS- $\alpha$ is that it satisfies Kelvin's circulation theorem (Holm et al. 1998a) while conserving energy and a form of potential vorticity (Holm, 1999), which requires that the line element of the flow on which the circulation is defined is advected along with the momentum. This constraint is ultimately responsible for appearance of the extra nonlinear term on the left side of (33). The terms involving $\mathbf{u}$ inside the modified pressure represent the kinetic energy of the flow (Foias et al., 2001).

The differences between (32) and the standard primitive equations distinguish the parts of (32) that comprise the AZ turbulence closure. Despite the similarity of (32) to LANS- $\alpha$, however, we must be cautious about extending the above physical interpretations to the AZ momentum equations. For example, the appearance of the double contraction term in (32) is a result of splitting the nonlinear stress in (3), and is not a part of the LANS- $\alpha$ framework. It is also unlikely that the Kelvin's circulation theorem is satisfied by AZ, due both to the presence of the double contraction term and the different velocities being advected and diffused. Some of these discrepancies are solved by considering the full nonlinear stress, not just the deformation part, which will be shown in Section 4

The analysis of AZ focuses on the parts of $\mathbf{S W}-\mathbf{W S}$ that affect the vorticity of the resolved flow, ignoring those parts which fall inside the gradient operator. Equation (32) shows that of the terms contributed by their closure, only the nonlinear term, $\nabla \mathbf{u}^{T} \cdot \mathbf{v}$, and double contraction term affect the vorticity. The analysis from Anstey and Zanna (2017) provides insight as to how these terms redistribute resolved energy locally, conserve energy in the domain-integrated sense, act as an enstrophy sink when vorticity gradients are sharpening, and are able to also strengthen vorticity gradients to maintain jets, at least for the 2D case. How each of these actions is controlled by the nonlinear and double-contraction terms individually has thus far not been explored, and is beyond the scope of this paper. The insight gained through such a study may be useful when considering the dynamical behavior and geometric interpretation (e.g. Waterman and Lilly 2015 ) of the nonlinear term in $\alpha$-models. 


\subsection{Memory}

The memory part of the stress tensor refers to the material derivative term that was neglected in AZ. Here we will complete the derivation as if this part was the candidate turbulence closure, using the stress tensor

$$
\boldsymbol{\sigma}=-p \mathbf{I}+2 \mu \mathbf{S}+2 \alpha_{1}\left[\frac{D \mathbf{S}}{D t}\right] .
$$

Taking the divergence of the memory term results in

$$
\begin{aligned}
\operatorname{div} \frac{D \mathbf{S}}{D t} & =\frac{1}{2} \frac{D \nabla^{2} \mathbf{u}}{D t}+\left(\nabla \mathbf{u}^{T} \cdot \nabla\right) \cdot \mathbf{S} \\
& =\frac{1}{2}\left[\frac{D \nabla^{2} \mathbf{u}}{D t}+\left(\nabla \mathbf{u}^{T} \cdot \nabla\right) \cdot \nabla \mathbf{u}+\left(\nabla \mathbf{u}^{T} \cdot \nabla\right) \cdot \nabla \mathbf{u}^{T}\right] .
\end{aligned}
$$

The term on the far right also appeared for the deformation part and obeys the identity (30), while the middle term can be expressed as

$$
\left(\nabla \mathbf{u}^{T} \cdot \nabla\right) \cdot \nabla \mathbf{u}=\nabla\left(\|\mathbf{S}\|^{2}-\frac{1}{2}\|\nabla \mathbf{u}\|^{2}\right) .
$$

Substituting (36) and these identities into (25) gives the momentum equations

$$
\frac{D \mathbf{v}}{D t}=-\frac{1}{\rho_{0}} \nabla\left(p-\rho_{0} \alpha\|\mathbf{S}\|^{2}+\rho_{0} \frac{\alpha}{2}\|\nabla \mathbf{u}\|^{2}\right)+\alpha \mathbf{S}: \mathbf{H}(\mathbf{u})+\nu \nabla^{2} \mathbf{u},
$$

where we see that subtracting the $\frac{D \nabla^{2} \mathbf{u}}{D t}$ term in (36) to the left side of (25) has led to an evolution equation for $\mathbf{v}$ rather than $\mathbf{u}$. The memory part of the nonlinear stress is thus associated with the material advection of v.

All terms in the memory part that lie inside the gradient operator do not affect the vorticity of the resolved flow, though they provide nonlocal contributions to the resolved energy. Identifying these issues may be important in parameterization design (e.g. Anstey and Zanna, 2017), as well as in understanding dynamics which contribute to the upscale cascade of kinetic energy (e.g. Kjellsson and Zanna, 2017). Interestingly, upon observing that the modified pressure terms in the deformation part (32) and LANS- $\alpha$ (33) are identical, we can now identify the memory part as being responsible for the difference between the modified pressure of the combined model (39) and LANS- $\alpha$. Like in the deformation part, the double-contraction term $\alpha \mathbf{S}: \mathbf{H}(\mathbf{u})$ appears as an extra forcing term on the right hand side, although with the opposite sign as in (32). The dissipation term, which is not associated with the nonlinear terms in the stress tensor (22), remains the same as for the deformation part.

\section{Combined memory and deformation}

We now return to the full expression (21) and show the model which results when both memory and deformation parts are combined. This amounts to adding the square bracketed terms in (26) and (36) together, and with identities (29), (30), and (37) the momentum equations become 


$$
\frac{D \mathbf{v}}{D t}+\nabla \mathbf{u}^{T} \cdot \mathbf{v}=-\frac{1}{\rho_{0}} \nabla\left(p-\frac{\rho_{0}}{2}\|\mathbf{u}\|^{2}-\rho_{0} \alpha\|\mathbf{S}\|^{2}\right)+\nu \nabla^{2} \mathbf{u} .
$$

The momentum equations for a second-grade Rivlin-Ericksen fluid (or "combined AZ" model) thus contain several key elements of the LANS- $\alpha$ model, including the material advection of $\mathbf{v}$ and the extra nonlinear term, $\nabla \mathbf{u}^{T} \cdot \mathbf{v}$. Comparison against the LANS- $\alpha$ equations, reprinted here from (33),

$$
\frac{D \mathbf{v}}{D t}+\nabla \mathbf{u}^{T} \cdot \mathbf{v}=-\frac{1}{\rho_{0}} \nabla\left(p-\frac{\rho_{0}}{2}\|\mathbf{u}\|^{2}-\rho_{0} \frac{\alpha}{2}\|\nabla \mathbf{u}\|^{2}\right)+\nu \nabla^{2} \mathbf{v}
$$

shows that the key differences between these models lie in the gradient operator and dissipative terms on the right side. In the LANS- $\alpha$ model, if one assumes homogeneous, isotropic Lagrangian fluctuations then

$$
\frac{1}{2} \overline{\left\|\mathbf{u}^{\prime}\right\|^{2}}=\frac{\alpha}{2}\|\nabla \mathbf{u}\|^{2}
$$

is interpreted as the (Eulerian) eddy kinetic energy (Holm, 1999). The presence of $\|\mathbf{S}\|$ instead of $\frac{1}{2}\|\nabla \mathbf{u}\|$ in (39) thus implies a different definition of the eddy kinetic energy between LANS- $\alpha$ and "combined AZ". Furthermore, for the particular choice of tensor norm in (18) we have

$$
\|\nabla \mathbf{u}\|^{2}=\|\mathbf{S}\|^{2}+\|\mathbf{W}\|^{2},
$$

implying that when $\|\mathbf{W}\| \geq\|\mathbf{S}\|$ the eddy kinetic energy of LANS- $\alpha$ is greater than that of the "combined AZ" model. The inverse of this statement also holds. We note that, because these terms lie inside the gradient operator in (39) and (40), they do not affect the vorticity or enstrophy themselves, so it is difficult to hypothesize about how the magnitude of $\|\mathbf{W}\|$ would tend to compare between the models. It is possible that the stronger dissipation term in LANS- $\alpha$ would tend to dissipate enstrophy more aggressively than "combined AZ", resulting in smaller $\|\mathbf{W}\|$ and less eddy kinetic energy. Further investigation of these ideas would benefit from a numerical implementation of $\mathrm{AZ}$ or its combined form, for which work is ongoing.

Analysis of (39) with regard to certain properties central to LANS- $\alpha$, such as satisfying Kelvin's circulation theorem and conservation of a form of potential vorticity (Holm, 1999), is beyond the scope of this paper. As it pertains to ocean eddy parameterization, it is unclear where the differences in the pressure and dissipation terms play a significant role in the dynamics in comparison with the modified advection terms on the left. However, recent work has shown that horizontal pressure gradients can play a significant role in upscale energy transfer (Kjellsson and Zanna, 2017), implying that subtle differences in the pressure may still be capable of imprinting on the large-scale flow. These topics are certainly worthy of future study.

\section{Conclusion}

In this article we have presented a derivation of the momentum equations for second-grade fluids of differential type using vector-tensor notation. A recently proposed mesoscale eddy closure by Anstey and Zanna (2017, AZ) can be obtained as part of this derivation. The results here extend that closure to 3D and include both the original "deformation" part as well as a "memory" term which was neglected in their analysis. We have presented versions of the momentum equations that result if one part or the other is 
neglected, which is meant to aid in their dynamical interpretation and to guide modellers who may wish to implement them separately.

An intriguing similarity exists between the second-grade fluid equations and the LANS- $\alpha$ turbulence model. This similarity has long been appreciated (e.g. Foias et al., 2001), but to this point remains relatively unexploited in terms of gaining physical insight about geophysical turbulence. In the context of the AZ closure, the connection with LANS- $\alpha$ establishes a link between different branches of developments in ocean eddy parameterizations and associated desirable properties of eddy-mean flow interaction. In particular, the dynamical behavior studied in AZ with regard to their closure can be identified with specific terms in the LANS- $\alpha$ model.

It is worth emphasizing that the AZ (and extended versions) and LANS- $\alpha$ turbulence models discussed here constitute a significant departure from the prevailing paradigm in large-scale ocean modelling, which is to rely on parameterizing individual processes rather than the entire turbulent continuum. An especially relevant example of such a process-based parameterization is that of Gent and McWilliams (Gent and McWilliams, 1990, hereafter GM) for mesoscale baroclinic instability. Despite the success of GM and its ubiquitous presence in large-scale ocean models, it has several notable shortcomings. For example, GM acts as a global sink of available potential energy, but it has no intrinsic mechanism to transfer this potential energy to the kinetic energy of the resolved flow. There are also open questions regarding how to handle this parameterization in the transition from non-eddying to eddy-permitting flow, where proposed solutions include either specifying a transport coefficient which is either explicitly resolution-dependent (e.g. Hallberg 2013) or scale- or flow-aware (e.g. Bachman et al., 2017; Porta Mana and Zanna, 2014). In essence, GM faces an issue of redundancy when the scale where potential-to-kinetic energy transfer becomes significant (the deformation radius) becomes resolved. The hierarchy of $\alpha$-models avoids this problem by shifting this energy transfer scale to lower wavenumbers (Holm and Wingate, 2005), effectively creating an artificial "deformation radius" that is always resolved. The consequences of this scale shifting with regard to the global oceanic flow, ventilation, and climate have not been well-explored, and a side-by-side comparison between models using GM, AZ and LANS- $\alpha$ has not yet been published.

The mathematical similarities between the different models shown here suggest a few possible avenues toward further research. The analysis performed in AZ yielded interesting new insights regarding the deformation terms in these models, so it seems natural that a similar analysis could be performed for the memory term. Such analyses might be helpful in establishing constraints to improve the numerical stability of these models, which was a significant challenge in previous studies of LANS- $\alpha$ (M. Hecht, personal communication). To date, neither the AZ scheme or its memory counterpart have been successfully implemented in a prognostic primitive equation ocean model, for which work is currently ongoing. Finally, some interesting theoretical questions linger about the differences between these models and their physical interpretation, such as the relationship between $\mathbf{S}, \nabla \mathbf{u}$, and the eddy (and total) kinetic energy. Other extensions of the $\alpha$-model hierarchy, such as the modified Leray- $\alpha$ model (e.g. Ilyin et al., 2006; Lunasin et al. , 2007, 2008) which switches the role of $\mathbf{u}$ and $\mathbf{v}$ in the extra nonlinear term $\nabla \mathbf{u}^{T} \cdot \mathbf{v}$, may also warrant exploration within the scope of ocean modeling. It is the authors' hope that the derivation presented here provokes further research into these nontraditional approaches to the ocean closure problem, such as LANS- $\alpha$ model and those proposed by Porta Mana and Zanna (2014) or AZ.

\section{Acknowledgements}

The work was supported in part by the NERC grant NE/K013548/1. The authors would like to thank Stephen Griffies for useful discussions on an earlier draft, as well as three anonymous reviewers whose comments greatly improved this manuscript. 


\section{References}

Andrews, D.G. and McIntyre, M.E., 1978. An exact theory of nonlinear waves on a Lagrangian-mean flow. Journal of Fluid Mechanics, 89(4), pp.609-646. doi:10.1017/S0022112078002773

Anstey, J.A. and Zanna, L., 2017. A deformation-based parametrization of ocean mesoscale eddy Reynolds stresses. Ocean Modelling, 112, pp.99-111. doi:10.1016/j.ocemod.2017.02.004

Bachman, S.D., Fox-Kemper, B. and Pearson, B., 2017. A scale-aware subgrid model for quasi-geostrophic turbulence. Journal of Geophysical Research: Oceans, 122(2), pp.1529-1554. doi:10.1002/2016JC012265

Berloff, P.S., 2005. Random-forcing model of the mesoscale oceanic eddies. Journal of Fluid Mechanics, 529, pp.71-95. doi:10.1017/S0022112005003393

Bhat, H.S., Fetecau, R.C., Marsden, J.E., Mohseni, K. and West, M., 2005. Lagrangian averaging for compressible fluids. Multiscale Modeling and Simulation, 3(4), pp.818-837. doi:10.1137/030601739

Callies, J. and Ferrari, R., 2013. Interpreting energy and tracer spectra of upper-ocean turbulence in the submesoscale range (1200 km). Journal of Physical Oceanography, 43(11), pp.2456-2474. doi:10.1175/JPOD-13-063.1

Camassa, R. and Holm, D.D., 1993. An integrable shallow water equation with peaked solitons. Physical Review Letters, 71(11), p.1661. doi:10.1103/PhysRevLett.71.1661

Capet, X., McWilliams, J.C., Molemaker, M.J. and Shchepetkin, A.F., 2008. Mesoscale to submesoscale transition in the California Current System. Part II: Frontal processes. Journal of Physical Oceanography, 38(1), pp.44-64. doi:10.1175/2007JPO3672.1

Chen, S., Foias, C., Holm, D.D., Olson, E., Titi, E.S. and Wynne, S., 1998. Camassa-Holm equations as a closure model for turbulent channel and pipe flow. Physical Review Letters, 81(24), p.5338. doi:10.1103/PhysRevLett.81.5338

Chen, S., Holm, D.D., Margolin, L.G. and Zhang, R., 1999. Direct numerical simulations of the Navier-Stokes alpha model. Physica D: Nonlinear Phenomena, 133(1), pp.66-83. doi:10.1016/S01672789(99)00099-8

Coleman, B.D. and Noll, W., 1960. An approximation theorem for functionals, with applications in continuum mechanics. Archive for Rational Mechanics and Analysis, 6(1), pp.355-370. doi:10.1007/BF00276168

Criminale, W.O., Ericksen, J.L. and Filbey, G.L., 1958. Steady shear flow of non-Newtonian fluids. Archive for Rational Mechanics and Analysis, 1(1), pp.410-417. doi:10.1007/BF00298018

Crow, S.C., 1968. Viscoelastic properties of fine-grained incompressible turbulence. Journal of Fluid Mechanics, 33(1), pp.1-20. doi:10.1017/S0022112068002314

Dill, E.H., 2006. Continuum mechanics: elasticity, plasticity, viscoelasticity. CRC press.

Duan, J. and Nadiga, B., 2007. Stochastic parameterization for large eddy simulation of geophysical flows. Proceedings of the American Mathematical Society, 135(4), pp.1187-1196. doi:10.1090/S0002-9939-0608631-X 
Dunn, J.E. and Fosdick, R.L., 1974. Thermodynamics, stability, and boundedness of fluids of complexity 2 and fluids of second grade. Archive for Rational Mechanics and Analysis, 56(3), pp.191-252. doi:10.1007/BF00280970

Dunn, J.E. and Rajagopal, K.R., 1995. Fluids of differential type: critical review and thermodynamic analysis. International Journal of Engineering Science, 33(5), pp.689-729. doi:10.1016/0020-7225(94)00078-X

Ericksen, J.L., 1956. Stress-deformation relations for solids. Canadian Journal of Physics, 34(2), pp.226227. doi:10.1139/p56-025

Foias, C., Holm, D.D. and Titi, E.S., 2001. The Navier-Stokes-alpha model of fluid turbulence. Physica D: Nonlinear Phenomena, 152, pp.505-519. doi:10.1016/S0167-2789(01)00191-9

Frederiksen, J.S. and Davies, A.G., 1997. Eddy viscosity and stochastic backscatter parameterizations on the sphere for atmospheric circulation models. Journal of the Atmospheric Sciences, 54(20), pp.2475-2492. doi:10.1175/1520-0469(1997)054<2475:EVASBP $>2.0 . C O ; 2$

Gent, P.R. and McWilliams, J.C., 1990. Isopycnal mixing in ocean circulation models. Journal of Physical Oceanography, 20(1), pp.150-155. doi: 10.1175/1520-0485(1990)020<0150:IMIOCM >2.0.CO;2

Gonzalez, O. and Stuart, A.M., 2008. A first course in continuum mechanics. Cambridge University Press.

Grooms, I. and Majda, A.J., 2013. Efficient stochastic superparameterization for geophysical turbulence. Proceedings of the National Academy of Sciences, 110(12), pp.4464-4469. doi:10.1073/pnas.1302548110

Grooms, I. and Zanna, L., 2017. A note on Toward a stochastic parameterization of ocean mesoscale eddies. Ocean Modelling, 113, pp.30-33. doi:10.1016/j.ocemod.2017.03.007

Gurtin, M.E., Fried, E. and Anand, L., 2010. The mechanics and thermodynamics of continua. Cambridge University Press.

Hallberg, R., 2013. Using a resolution function to regulate parameterizations of oceanic mesoscale eddy effects. Ocean Modelling, 72, pp.92-103. doi:10.1016/j.ocemod.2013.08.007

Hecht, M.W., Holm, D.D., Petersen, M.R. and Wingate, B.A., 2008a. Implementation of the LANS- $\alpha$ turbulence model in a primitive equation ocean model. Journal of Computational Physics, 227(11), pp.56915716. doi:10.1016/j.jcp.2008.02.018

Hecht, M.W., Holm, D.D., Petersen, M.R. and Wingate, B.A., 2008b. The LANS- $\alpha$ and Leray turbulence parameterizations in primitive equation ocean modeling. Journal of Physics A: Mathematical and Theoretical, 41(34), p.344009. doi:10.1088/1751-8113/41/34/344009

Holm, D.D., 1999. Fluctuation effects on 3D Lagrangian mean and Eulerian mean fluid motion. Physica D: Nonlinear Phenomena, 133(1), pp.215-269. doi:10.1016/S0167-2789(99)00093-7

Holm, D.D., 2002. Lagrangian averages, averaged Lagrangians, and the mean effects of fluctuations in fluid dynamics. Chaos: An Interdisciplinary Journal of Nonlinear Science, 12(2), pp.518-530. doi:10.1063/1.1460941

Holm, D.D., Jeffery, C., Kurien, S., Livescu, D., Taylor, M.A. and Wingate, B.A., 2005. The LANS- $\alpha$ model for computing turbulence. Los Alamos Science, 29, pp.152-171. 
Holm, D.D., Marsden, J.E. and Ratiu, T.S., 1998a. Euler-Poincaré models of ideal fluids with nonlinear dispersion. Physical Review Letters, 80(19), p.4173. doi:10.1103/PhysRevLett.80.4173

Holm, D.D., Marsden, J.E. and Ratiu, T.S., 1998b. The Euler-Poincaré equations and semidirect products with applications to continuum theories. Advances in Mathematics, 137(1), pp.1-81. doi:10.1006/aima.1998.1721

Holm, D.D. and Nadiga, B.T., 2003. Modeling mesoscale turbulence in the barotropic doublegyre circulation. Journal of Physical Oceanography, 33(11), pp.2355-2365. doi:10.1175/15200485(2003)033<2355:MMTITB >2.0.CO;2

Holm, D.D., Putkaradze, V., Weidman, P.D. and Wingate, B.A., 2003. Boundary effects on exact solutions of the Lagrangian-averaged Navier-Stokes- $\alpha$ equations. Journal of Statistical Physics, 113(5), pp.841-854. doi:10.1023/A:1027364720090

Holm, D.D. and Wingate, B.A., 2005. Baroclinic instabilities of the two-layer quasigeostrophic alpha model. Journal of Physical Oceanography, 35(7), pp.1287-1296. doi:10.1175/JPO2741.1

Ilyin, A.A., Lunasin, E.M. and Titi, E.S., 2006. A modified-Leray- $\alpha$ subgrid scale model of turbulence. Nonlinearity, 19(4), p.879. doi:10.1088/0951-7715/19/4/006

Jansen, M.F. and Held, I.M., 2014. Parameterizing subgrid-scale eddy effects using energetically consistent backscatter. Ocean Modelling, 80, pp.36-48. doi:10.1016/j.ocemod.2014.06.002

Jansen, M.F., Held, I.M., Adcroft, A. and Hallberg, R., 2015. Energy budget-based backscatter in an eddy permitting primitive equation model. Ocean Modelling, 94, pp.15-26. doi:10.1016/j.ocemod.2015.07.015

Kitsios, V., Frederiksen, J.S. and Zidikheri, M.J., 2013. Subgrid parameterisation of the eddymeanfield interactions in a baroclinic quasi-geostrophic ocean. ANZIAM Journal, 54, pp.459-475. doi:10.21914/anziamj.v54i0.6309

Klein, P., Hua, B.L., Lapeyre, G., Capet, X., Le Gentil, S. and Sasaki, H., 2008. Upper ocean turbulence from high-resolution 3D simulations. Journal of Physical Oceanography, 38(8), pp.1748-1763. doi:10.1175/2007JPO3773.1

Kjellsson, J. and Zanna, L., 2017. The Impact of Horizontal Resolution on Energy Transfers in Global Ocean Models. Fluids, 2(3), p.45. doi:10.3390/fluids2030045

Lumley, J.L., 1970. Toward a turbulent constitutive relation. Journal of Fluid Mechanics, 41(2), pp.413-434. doi:10.1017/S0022112070000678

Lunasin, E., Kurien, S., Taylor, M.A. and Titi, E.S., 2007. A study of the Navier-Stokes- $\alpha$ model for twodimensional turbulence. Journal of Turbulence, (8), p.N30. doi:10.1080/14685240701439403

Lunasin, E., Kurien, S. and Titi, E.S., 2008. Spectral scaling of the Leray- $\alpha$ model for two-dimensional turbulence. Journal of Physics A: Mathematical and Theoretical, 41(34), p.344014. doi:10.1088/1751$8113 / 41 / 34 / 344014$

Marsden, J.E. and Shkoller, S., 2001. Global well-posedness for the Lagrangian averaged Navier-Stokes (LANS- $\alpha$ ) equations on bounded domains. Philosophical Transactions of the Royal Society of London A: Mathematical, Physical and Engineering Sciences, 359(1784), pp.1449-1468. doi:10.1098/rsta.2001.0852 
Marsden, J.E. and Shkoller, S., 2003. The anisotropic Lagrangian averaged Euler and Navier-Stokes equations. Archive for Rational Mechanics and Analysis, 166(1), pp.27-46. doi:10.1007/s00205-002-0207-8

Meneveau, C. and Katz, J., 2000. Scale-invariance and turbulence models for large-eddy simulation. Annual Review of Fluid Mechanics, 32(1), pp.1-32. doi:10.1146/annurev.fluid.32.1.1

Pearson, B., Fox-Kemper, B., Bachman, S.D., and Bryan, F.O., 2017. Evaluation of scale-aware subgrid mesoscale eddy models in a global eddy-rich model. Ocean Modelling, 115, pp.42-58. doi:10.1016/j.ocemod.2017.05.007

Petersen, M.R., Hecht, M.W. and Wingate, B.A., 2008. Efficient form of the LANS- $\alpha$ turbulence model in a primitive-equation ocean model. Journal of Computational Physics, 227(11), pp.5717-5735. doi:10.1016/j.jcp.2008.02.017

Porta Mana, P.G.L. and Zanna, L., 2014. Toward a stochastic parameterization of ocean mesoscale eddies. Ocean Modelling, 79, pp.1-20. doi:10.1016/j.ocemod.2014.04.002

Rivlin, R.S., 1957. The relation between the flow of non-Newtonian fluids and turbulent Newtonian fluids. Quarterly of Applied Mathematics, 15(2), pp.212-215. doi:10.1007/978-1-4612-2416-7_128

Rivlin, R.S. and Ericksen, J.L., 1955. Stress-deformation relations for isotropic materials. Journal of Rational Mechanics and Analysis, 4, pp.323-425. doi:10.1007/978-1-4612-2416-7_61

Rocha, C.B., Chereskin, T.K., Gille, S.T. and Menemenlis, D., 2016. Mesoscale to submesoscale wavenumber spectra in Drake Passage. Journal of Physical Oceanography, 46(2), pp.601-620. doi:10.1175/JPO-D$15-0087.1$

Sasaki, H. and Klein, P., 2012. SSH wavenumber spectra in the North Pacific from a high-resolution realistic simulation. Journal of Physical Oceanography, 42(7), pp.1233-1241. doi.org/10.1175/JPO-D-11-0180.1

Scott, R.B. and Arbic, B.K., 2007. Spectral energy fluxes in geostrophic turbulence: Implications for ocean energetics. Journal of physical oceanography, 37(3), pp.673-688. doi:10.1175/JPO3027.1

Scott, R.B. and Wang, F., 2005. Direct evidence of an oceanic inverse kinetic energy cascade from satellite altimetry. Journal of Physical Oceanography, 35(9), pp.1650-1666. doi:10.1175/JPO2771.1

Stammer, D., 1997. Global characteristics of ocean variability estimated from regional TOPEX/POSEIDON altimeter measurements. Journal of Physical Oceanography, 27(8), pp.1743-1769. doi:10.1175/15200485(1997)027<1743:GCOOVE > 2.0.CO;2

Wang, D.P., Flagg, C.N., Donohue, K. and Rossby, H.T., 2010. Wavenumber spectrum in the Gulf Stream from shipboard ADCP observations and comparison with altimetry measurements. Journal of Physical Oceanography, 40(4), pp.840-844. doi:10.1175/2009JPO4330.1

Waterman, S. and Lilly, J.M., 2015. Geometric decomposition of eddy feedbacks in barotropic systems. Journal of Physical Oceanography, 45(4), pp.1009-1024. doi:10.1175/JPO-D-14-0177.1

Zanna, L., Porta Mana, P.G.L., Anstey, J., David, T. and Bolton, T., 2017. Scale-aware deterministic and stochastic parametrizations of eddy-mean flow interaction. Ocean Modelling, 111, pp.66-80. doi:10.1016/j.ocemod.2017.01.004 